\title{
Climate, orography and scale controls on flood frequency in Triveneto (Italy)
}

\author{
Simone Persiano ${ }^{1}$, Attilio Castellarin ${ }^{1}$, Jose Luis Salinas ${ }^{2}$, Alessio Domeneghetti ${ }^{1}$, and Armando Brath ${ }^{1}$ \\ ${ }^{1}$ Department DICAM, School of Civil Engineering, University of Bologna, Bologna, Italy \\ ${ }^{2}$ Institute of Hydraulic Engineering and Water Resources Management, Vienna University of Technology, \\ Vienna, Austria \\ Correspondence to: Simone Persiano (simone.persiano2@unibo.it)
}

Published: 12 May 2016

\begin{abstract}
The growing concern about the possible effects of climate change on flood frequency regime is leading Authorities to review previously proposed reference procedures for design-flood estimation, such as national flood frequency models. Our study focuses on Triveneto, a broad geographical region in North-eastern Italy. A reference procedure for design flood estimation in Triveneto is available from the Italian NCR research project "VA.PI.", which considered Triveneto as a single homogeneous region and developed a regional model using annual maximum series (AMS) of peak discharges that were collected up to the 1980s by the former Italian Hydrometeorological Service. We consider a very detailed AMS database that we recently compiled for 76 catchments located in Triveneto. All 76 study catchments are characterized in terms of several geomorphologic and climatic descriptors. The objective of our study is threefold: (1) to inspect climatic and scale controls on flood frequency regime; (2) to verify the possible presence of changes in flood frequency regime by looking at changes in time of regional L-moments of annual maximum floods; (3) to develop an updated reference procedure for design flood estimation in Triveneto by using a focused-pooling approach (i.e. Region of Influence, RoI). Our study leads to the following conclusions: (1) climatic and scale controls on flood frequency regime in Triveneto are similar to the controls that were recently found in Europe; (2) a single year characterized by extreme floods can have a remarkable influence on regional flood frequency models and analyses for detecting possible changes in flood frequency regime; (3) no significant change was detected in the flood frequency regime, yet an update of the existing reference procedure for design flood estimation is highly recommended and we propose the RoI approach for properly representing climate and scale controls on flood frequency in Triveneto, which cannot be regarded as a single homogeneous region.
\end{abstract}

\section{Introduction}

One of the most common tasks in hydrology is to produce an accurate estimation of the design flood at ungauged or sparsely gauged river cross-sections (see e.g. Salinas et al., 2014, and references therein). This task is often addressed by means of regional flood frequency analysis by collecting flood data from gauged basins which are supposed to be hydrologically similar to the target (ungauged or sparsely gauged) basin (see Hosking and Wallis, 1993). Our study focuses on Triveneto, a broad mountainous geographical area in North-eastern Italy consisting of the administrative districts of Trentino-Alto Adige, Veneto and Friuli-Venezia Giu- lia; the study area counts numerous dams that routinely undergo hydrologic and hydraulic risk assessments. A reference procedure for design flood estimation in Triveneto is available from the Italian NCR research project "VA.PI.", which developed an index-flood regional model based upon annual maximum series (AMS) of peak discharges that were collected up to the 1980 s. We consider a very detailed AMS database that we recently compiled for 76 catchments located in Triveneto. Our annual flood sequences include historical data together with more recent data, and the data sets spans from 1913 to 2012. 


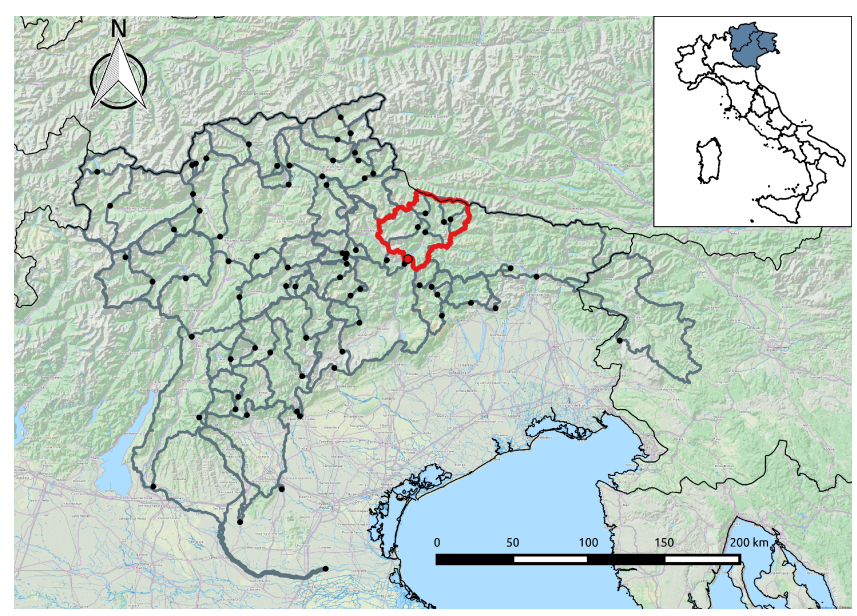

Figure 1. Location of the 76 Triveneto gauging stations (black points) considered in the present study. Grey lines represent catchments boundaries. Pieve di Cadore, an example target basin, is highlighted in red.

The objective of our study is threefold: (1) to check whether climatic and scale controls on flood frequency regime in Triveneto are similar to the controls that were recently found in Europe; (2) to verify the possible presence of changes in flood frequency regime by looking at changes in time of regional L-moments of annual maximum floods (see Hosking and Wallis, 1993, for an introduction to Lmoments); (3) to develop an updated reference procedure for design flood estimation in Triveneto by using a focusedpooling approach (i.e. Region of Influence, RoI; see Burn, 1990).

\section{Area and MAP control on regional flood frequency distribution}

The selection of the most suitable regional parent distribution is a key aspect in any regional flood frequency analysis. The scientific literature recommends using the L-moment ratio diagrams for addressing this task (see e.g. Hosking and Wallis, 1993). Because of the unavailability or high uncertainty of sample statistics for ungauged or poorly gauged regions, many studies have focused on the relationships between sample L-moments and catchment descriptors. In particular, a recent study by Salinas et al. (2014) on a data set of annual maximum series (AMS) of peak flow from a total of 813 catchments (in Austria, Italy and Slovakia) shows the great importance of incorporating into regional models information on mean annual precipitation (MAP) and basin area as surrogates of climate and scale controls. The first main aim of our study is to check whether climatic and scale controls on flood frequency regime in Triveneto are similar to the controls that were recently found in Europe by Salinas et al. (2014).

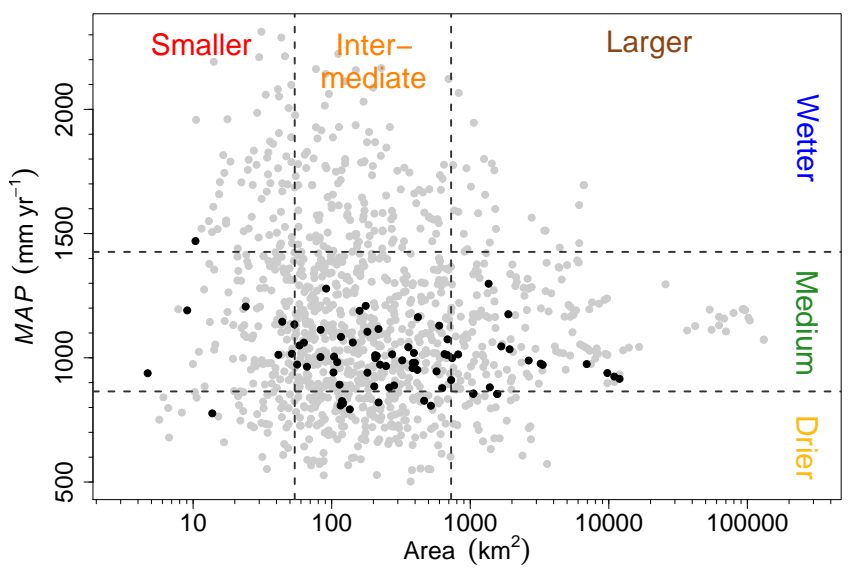

Figure 2. Catchment characteristics of the Triveneto data set (black points). Grey points represent the Austrian, Slovakian and Italian data sets considered in Salinas et al. (2014). Black-dashed lines identify the subdivision into the six subsets based on the 20 and $80 \%$ quantiles of the catchments descriptor values for the European data sets.

The study region consists of 76 catchments (see Fig. 1) with minimum, mean and maximum length of observed AMS equal to 5, 31 and 87 years, in this order. To reduce the effects of sampling variability when estimating higher order L-moments (see e.g. Viglione, 2010), the information from each site was weighted proportionally to the site record length (Hosking and Wallis, 1993) during all regionalization phases. For comparison purposes, the data set was divided into the same six subsets identified in Salinas et al. (2014): smaller (area $<55 \mathrm{~km}^{2}$ ), intermediate $\left(55 \mathrm{~km}^{2}<\right.$ area $\left.<730 \mathrm{~km}^{2}\right)$ and larger $\left(\right.$ area $\left.>730 \mathrm{~km}^{2}\right)$ catchments, drier $\left(\right.$ MAP $<860 \mathrm{~mm} \mathrm{yr}^{-1}$ ), medium $\left(860 \mathrm{~mm} \mathrm{yr}^{-1}<\mathrm{MAP}<1420 \mathrm{~mm} \mathrm{yr}^{-1}\right.$ ) and wetter $\left(\mathrm{MAP}>1420 \mathrm{~mm} \mathrm{yr}^{-1}\right.$ ) catchments. It is evident that the majority of the Triveneto catchments (black points in Fig. 2) belong to the central subset. So, in order to verify the impact of catchment size and MAP on the L-moment ratios for the Triveneto regional parent distribution, we considered only the two subsets associated with intermediate site and medium MAP. For each one of them, the two L-moment ratio diagrams $\mathrm{L}-\mathrm{C} v-\mathrm{L}-\mathrm{Cs}$ and $\mathrm{L}-\mathrm{Ck}-\mathrm{L}-\mathrm{Cs}$ were plotted, including the theoretical lines for the most common twoand three-parameter distributions, respectively (see Fig. 3). Similarly to Salinas et al. (2014), the points drawn in the diagrams represent the record length weighted moving average (WMA) values of the corresponding sample L-moments and their colour intensity is proportional to the mean value of the descriptor not used for the stratification. WMA was computed for a window including the 35 most similar catchments in terms of the evaluated descriptor (i.e. area or MAP). 

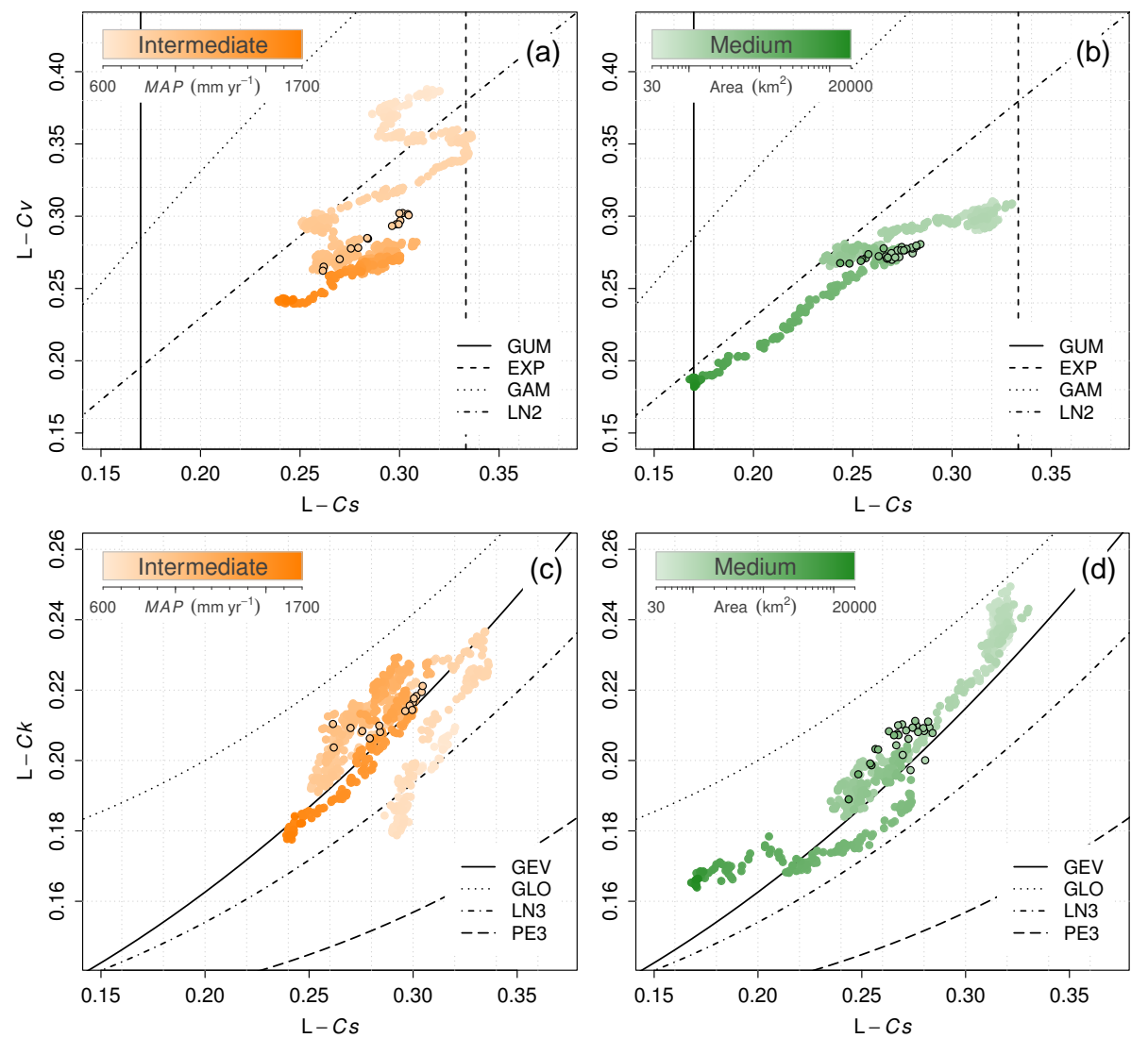

Figure 3. L-moment ratio diagrams for the subsets defined by intermediate area (a, c) and medium MAP (b, d). Each point represents a record length weighted moving average (WMA) of L-Cv $(\mathbf{a}, \mathbf{b})$ or L-Ck $(\mathbf{c}, \mathbf{d})$ against the corresponding values of L-Cs and the colour intensity is proportional to the catchment descriptor of interest (area or MAP); WMAs are computed for either 35 Triveneto basins (black-border) or 70 European basins (no-border) having similar area or MAP value.

Figure 3 clearly shows the significant superimposition of Triveneto WMAs to WMAs obtained by Salinas et al. (2014). Figure $3 \mathrm{a}$ and $\mathrm{b}$ confirm that sample $\mathrm{L}-\mathrm{Cv}$ and $\mathrm{L}-\mathrm{Cs}$ are poorly described by the most common two-parameter distributions. On the other hand, Fig. 3c shows how medium-sized (intermediate) catchments associate with high to medium MAP are well described by the Generalized Extrem Value (GEV) distribution, and Fig. 3d clearly reports the same behaviour for medium MAP catchments. The strong control of area and MAP on regional L-moments (i.e. WMAs) is also confirmed by the intensity of gradation, which increases with decreasing L-moment ratios values for all the considered cases: average L-moment ratio values are higher for smaller than larger catchments and for drier than wetter ones.

\section{Changes in flood frequency}

The routinely hydrological and hydraulic risk assessments of dams located in Triveneto are related to the growing concern about the possible effects of climate change on the region flood-frequency regime. A second main objective of this study is therefore to verify the possible presence of changes in flood frequency regime by looking at the variability of regional L-moments of annual maximum floods in time. In fact, according to Beniston (2012), Alps are a particularly sensitive context for climate change, and Castellarin and Pistocchi (2012) detected noticeable variations in the frequency regime of AMF in the last five decades in the Swiss Alps, with significant and abrupt changes of the mean flood, with change-point years identified between 1942 and 1977. Changes in the flood frequency regime for alpine Swiss catchments are also highlighted in Allamano et al. (2009b) and Schmocker-Fackel and Naef (2010), from the 1960s and the 1970 s, respectively.

In this context, we decided to check the presence of changes in the frequency regime of AMS in Triveneto with reference to 1965, by dividing the total AMS sample into two sub-samples: PRE1965 (all years before 1965, for a total of 1217 station-years of data) and POST1965 (all years from 1965, included; 1148 station-years of data). It is important to highlight that POST1965 sub-sample includes 1966, which was characterized by exceptionally heavy floods in Triveneto, and Italy in general. Year 1966 is well-known for the 4-5 November floods in Florence, but the entire autumn 

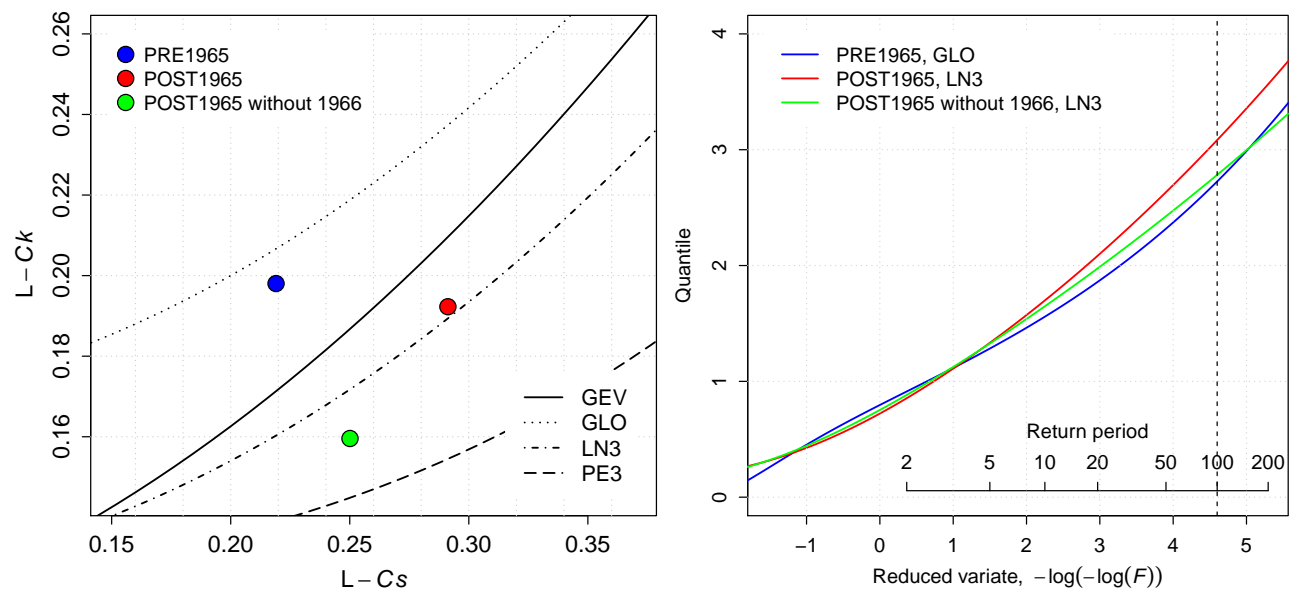

Figure 4. Left: L-moment ratio diagram for three-parameter distributions. Blue, red and green points represent the record length weighted average of L-Cv and L-Cs in Triveneto for PRE1965, POST1965 and POST1965 without 1966 sub-samples, in this order. Right: dimensionless growth-curves associated with the above mentioned three sub-samples. The choice of the corresponding three-parameter distribution was made by applying Hosking and Wallis test (see Hosking and Wallis, 1993).

of 1966 was particularly difficult for North-East Italian regions: Triveneto counted 87 deaths and about 42000 displaced people (data from the Italian NCR). Hence, we decided to test the importance of this year in the flood frequency regime, by analysing a further sub-sample, obtained by removing AMF of 1966 from the POST1965 sub-sample (1117 station-years of data).

For each of the considered three sub-samples we plotted the record length weighted average of $\mathrm{L}-\mathrm{Cv}$ and $\mathrm{L}$ $\mathrm{Cs}$ in the corresponding diagram and estimated the associated regional three-parameter distribution (see Fig. 4). The assessment of the best fitting statistical models was done through a visual inspection (observing the distance between the averaged sample L-moment ratios and the theoretical lines), and confirmed by the Hosking and Wallis goodnessof-the-fit test (see Hosking and Wallis, 1993). As reported in Fig. 4, left, the differences shown by the three subsamples are remarkable: whereas POST1965 sub-sample lays near the generalized logistic (GLO) theoretical curve, POST1965 and POST1965 without 1966 are associated with a three-parameter log-normal (LN3) distribution. Observing the shape of the dimensionless growth curve (see Fig. 4, right) differences are clear: for the 100-year return period, POST1965 shows a higher quantile than the other two subsamples. In particular, it is important to highlight the high similarity between PRE1965 and POST1965 without 1966. This is emphasised by observing that the important differences observed between POST1965 and POST1965 without 1966 are due to 31 station-years of data only (the difference between 1148 and 1117 station-years of data). This behaviour shows how a single exceptional year characterized by extreme floods can heavily influence the observed flood frequency regime and therefore the results of the statistical analysis performed to detect changes.

\section{Region of Influence approach}

It is important to highlight that the dimensionless growthcurves in Fig. 4 do not refer to a homogeneous regional sample. In fact, although Triveneto was considered as a single homogeneous region by the Italian NCR research project "VA.PI." (by using AMS collected up to the 1980s, see Villi and Bacchi, 2001), the significant update done in our study showed that the hypothesis of homogeneity does not hold for Triveneto. The Hosking and Wallis test (see Hosking and Wallis, 1993) indicates for the study region a high heterogeneity degree $\left(H_{1}>8\right.$, when the threshold value of $H_{1}$ for acceptably homogeneous regions is 1 ) for the overall Triveneto sample, and also for the three sub-samples shown in Fig. 4 ( $H_{1}$ is approximately 4 and 9 for PRE1965 and the two POST1965 sub-samples, respectively).

The third and last main objective of our study is to update the Triveneto reference procedure for flood frequency estimation. Therefore we tested and applied the so called Region of Influence (RoI) approach (see Burn, 1990, and Zrinji and Burn, 1996), which replaces the idea of homogeneous regions consisting of contiguous and geographically identifiable regions with the most general idea of homogeneous groups of basins with similar hydrological behaviour, which may or may not be geographically close to each other. The RoI approach delineates homogeneous pooling-groups for a given target site referring to a minimum amount of information in terms of climatic and geomorphological descriptors of the basins that strongly influence the flood frequency regime at regional scale. In our study we consider the following descriptors: area and MAP (whose strong influence on the flood frequency regime has been confirmed in this study), mean and minimum elevation and latitude and longitude of catchment centroid. In particular, it is very important to con- 

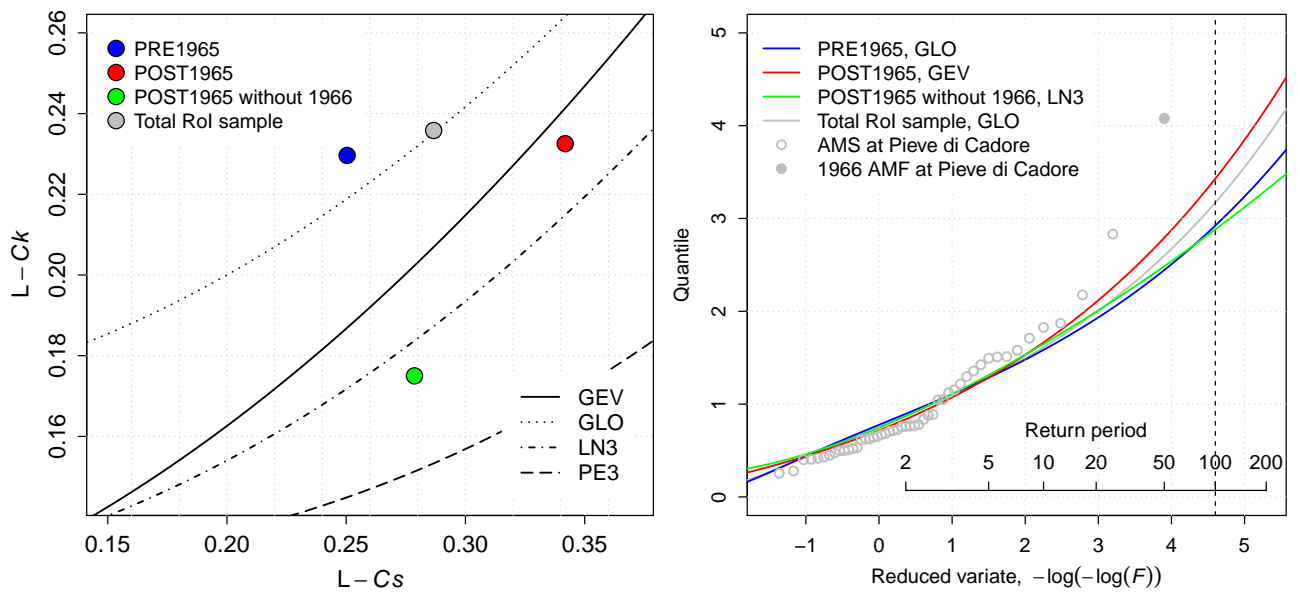

Figure 5. Left: L-moment ratio diagram for three-parameter distributions. Blue, red, green and grey points represent the record length weighted average of L-Cv and L-Cs for PRE1965, POST1965 and POST1965 without 1966 RoI sub-samples and total RoI sample, in this order. Right: dimensionless growth-curves associated with the above mentioned four sub-samples. The choice of the corresponding threeparameter distribution was made by applying Hosking and Wallis test (see Hosking and Wallis, 1993). Grey points indicate the empirical growth-curve for Pieve di Cadore (see Fig. 1, the full coloured point is the 1966 annual maximum flood).

sider catchment elevation as a descriptor of the orographic effect on the flood frequency regime (see e.g. Allamano et al., 2009a). The Euclidean distance in the 6-dimensional descriptor space was used as measure of hydrological dissimilarity (see e.g. Zrinji and Burn, 1996), where the same weight has been assigned to each of the mentioned descriptors.

The RoI approach arranges catchments according to their dissimilarity with the target site, pooling together only the most similar ones. The number of catchments to include in the RoI is determined by considering the return period of the design flood $T$ : according to the " $5 T$ rule" proposed by Jakob et al. (1999), a number of station-years of annual maxima $N \geq 5 T$ is required to get a reliable estimation of the $T$-year flood ( $T$-quantile). Therefore the RoI sample consists of a pooling-group of catchments that are the most hydrologically similar to the target site and whose overall station-years of data sums up to $5 T$ (i.e. 500 station-years of data for $T=$ 100 years).

We report herein an application example for the Pieve di Cadore dam, for which a 49 AMS (from 1951 to 1999) has been compiled from inflow series to the artificial reservoir. The RoI sample consists of 16 catchments, for a total of 555 observations, which enable one to get a reliable prediction of the 100-year quantile according to the " $5 T$ rule". We can observe the significant advantage of the RoI approach in terms of homogeneity: the heterogeneity associated with the RoI sample $\left(H_{1} \simeq 1.48\right)$ is much lower than that of the total Triveneto sample. Therefore the grey point in Fig. 5, left, and the corresponding RoI dimensionless growth-curve (grey line in in Fig. 5, right) are characteristics of a regional parent distribution that is suitable for predicting dimensionless flood quantiles up to $T=100$ years for Pieve di Cadore. Similarly to what was done for Triveneto, we checked the presence of changes in the frequency regime by splitting the total RoI sample into the three sub-samples PRE1965 (343 years of data), POST1965 (212 years of data) and POST1965 without 1966 (208 years of data). Figure 5 shows the remarkable differences between the three sub-samples: their shapes are associated to GLO, GEV (generalized extreme value) and LN3 distributions, respectively. In particular, the superimposition of PRE1965 and POST1965 without 1966, and the significant difference between the two POST1965 sub-samples, highlight again the great influence of a single exceptional year (1966, in our study) on the estimation of the flood frequency regime. This difference is even more striking in this case since it is due to 4 station-years of data.

\section{Conclusions}

Our study confirmed the value of including climatic and physiographic information in statistical regionalization. Catchment descriptors as mean annual precipitation (MAP), basin area, mean and minimum elevation and catchment location can be used as surrogates of climate and scale controls. In particular, our study shows that climatic and scale controls on flood frequency regime in Triveneto are similar to the controls that were recently found in Europe by Salinas et al. (2014): the regional flood frequency regime of medium-sized catchments associated with high to medium MAP is well described by the GEV distribution and average L-moment ratio values are higher for smaller than larger catchments and for drier than wetter ones.

By splitting the Triveneto sample into two distinct subsamples (i.e. before and after 1965) and then removing the 1966 year from the second one, we observe very similar conditions in terms of flood frequency regime, highlighting the 
remarkable influence that a single exceptional year (1966) can have in the representation of flood frequency regime.

Finally, our update of Triveneto AMS data set falsified the main assumption of the current reference procedure for design-flood estimation in Triveneto (see Villi and Bacchi, 2001), showing that the study region is not homogeneous in terms of flood frequency regime. Therefore, we developed an updated reference procedure by using the Region of Influence (RoI) approach. The RoI approach delineates homogeneous pooling-groups of sites for any given target site referring to selected climatic and geomorphological descriptors that are particularly relevant for describing regional flood frequency (e.g. the ones listed above). We observe that the regional sample obtained through the RoI approach for Pieve di Cadore (used as an example) is characterized by a homogeneity degree which is much higher than the Triveneto one, and shows no differences between past and present flood frequency regime, confirming again the great importance of the flood information collected in the exceptional year 1966 for correctly representing the site flood frequency distribution.

Acknowledgements. The study is part of the research activities carried out by the working group: Anthropogenic and Climatic Controls on WateR AvailabilitY (ACCuRAcY) of Panta Rhei - Everything Flows Change in Hydrology and Society (IAHS Scientific Decade 2013-2022).

\section{References}

Allamano, P., Claps, P., and Laio, F.: An analytical model of the effects of catchment elevation on the flood frequency distribution, Water Resour. Res., 45, W01402, doi:10.1029/2007WR006658, 2009a.

Allamano, P., Claps, P., and Laio, F.: Global warming increases flood risk in mountainous areas, Geophys. Res. Lett., 36, L24404, doi:10.1029/2009GL041395, 2009b.

Beniston, M.: Impacts of climatic change on water and associated economic activities in the Swiss Alps, J. Hydrology, 412-413, 291-296, doi:10.1016/j.jhydrol.2010.06.046, 2012.

Burn, D. H.: Evaluation of regional flood frequency analysis with a region of influence approach, Water Resour. Res., 26, 2257, doi:10.1029/WR026i010p02257, 1990.

Castellarin, A. and Pistocchi, A.: An analysis of change in alpine annual maximum discharges: implications for the selection of design discharges, Hydrol. Process., 26, 1517-1526, doi:10.1002/hyp.8249, 2012.

Hosking, J. R. M. and Wallis, J. R.: Some statistics useful in regional frequency analysis, Water Resour. Res., 29, 271-281, doi:10.1029/92WR01980, 1993.

Jakob, D., Reed, D. W., and Robson, A. J.: Choosing a poolinggroup, in: Flood Estimation Handbook, Institute of Hydrology, Wallingford, UK, Volume 3, 1999.

Salinas, J. L., Castellarin, A., Kohnová, S., and Kjeldsen, T. R.: Regional parent flood frequency distributions in Europe - Part 2: Climate and scale controls, Hydrol. Earth Syst. Sci., 18, 43914401, doi:10.5194/hess-18-4391-2014, 2014.

Schmocker-Fackel, P. and Naef, F.: Changes in flood frequencies in Switzerland since 1500, Hydrol. Earth Syst. Sci., 14, 1581-1594, doi:10.5194/hess-14-1581-2010, 2010.

Viglione, A.: Confidence intervals for the coefficient of L-variation in hydrological applications, Hydrol. Earth Syst. Sci., 14, 22292242, doi:10.5194/hess-14-2229-2010, 2010.

Villi, V. and Bacchi, B.: Valutazione delle piene nel Triveneto, CNR-GNDCI, Padova-Brescia, Italia, 2001.

Zrinji, Z. and Burn, D. H.: Regional Flood Frequency with Hierarchical Region of Influence, J. Water Res. Pl.-ASCE, 122, 245252, 1996. 